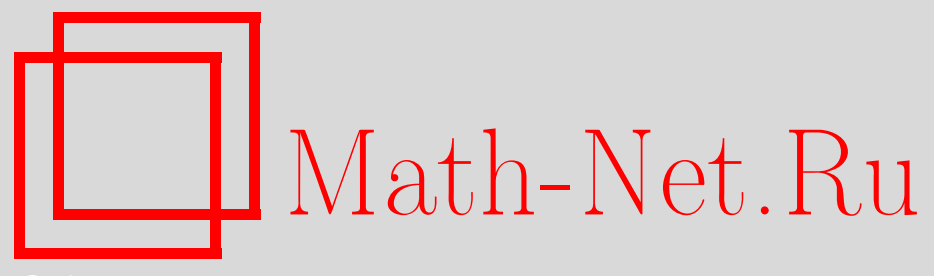

В. В. Кучеренко, А. В. Кривко, О теореме существования для гиперболических систем с точкой смены кратности порядка большего или равного трем, Матем. заметки, 2009, том 85, выпуск 1, 139-143

DOI: https://doi.org/10.4213/mzm6588

Использование Общероссийского математического портала Math-Net.Ru подразумевает, что вы прочитали и согласны с пользовательским соглашением http://www . mathnet.ru/rus/agreement

Параметры загрузки:

IP : 52.90 .164 .192

26 апреля 2023 г., 13:24:20

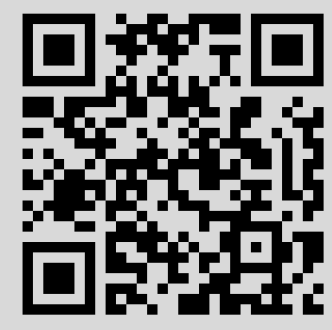




\section{О теореме существования для гиперболических систем с точкой смены кратности порядка большего или равного трем}

\section{В. В. Кучеренко, А. В. Кривко}

В данной работе мы рассматриваем строго гиперболические системы, т.е. системы, для которых задача Коши корректна при любых младших членах [1]. В работе [2] было показано, что из строгой гиперболичности следует, что в точках смены кратности главный символ строго гиперболической системы может иметь жорданову клетку не более второго порядка. В работе [3] рассматривается теорема существования для строго гиперболических систем с неинволютивными характеристиками и точками смены кратности порядка большего или равного трем, у которых главные символы имеют жордановые клетки второго порядка. В данной работе мы доказываем, что при изменении условий, приведенных в работе [3], задача Коши становится некорректной.

Предположим, что главный символ вещественной гиперболической системы первого порядка имеет вид

$$
A\left(x^{\prime}, \eta, \xi\right)=\eta I+T\left(x^{\prime}, \xi\right)\left\|b_{i j}^{0}\left(x^{\prime}, \xi\right)\right\| T^{-1}\left(x^{\prime}, \xi\right),
$$

где $I$ - это единичная $((r+2) \times(r+2))$-матрица, $x^{\prime}=(t, x)$, символы $T, T^{-1} \in S^{0}\left(\mathbb{R}_{x^{\prime}, \xi}^{2 n+1}\right)$, $b_{i j}^{0} \in S^{1}\left(\mathbb{R}_{x^{\prime}, \xi}^{2 n+1}\right)$ и

$$
\left\|b_{i j}^{0}\left(x^{\prime}, \xi\right)\right\|=\left(\begin{array}{ccc}
\lambda_{1} I_{r} & 0 & 0 \\
0 & \lambda_{2} & d \\
0 & 0 & \lambda_{3}
\end{array}\right)
$$

где $I_{r}-$ это единичная $(r \times r)$-матрица и

$$
\inf _{x^{\prime} \in \mathbb{R}_{x^{\prime}}^{n+1},|\xi|=1} d>0 .
$$

Обозначим через $A_{c}\left(x^{\prime}, \eta, \xi\right)$ полный символ этой системы первого порядка. В этом случае из анализа псевдодифференциальных операторов (ПДО) следует, что сумма ПДО операторов вида

$$
T^{-1}\left(x^{\prime},-i \frac{\partial}{\partial x}\right) A_{c}\left(x^{\prime},-i \frac{\partial}{\partial t},-i \frac{\partial}{\partial x}\right) T\left(x^{\prime},-i \frac{\partial}{\partial x}\right)+(-i) T^{-1}\left(x^{\prime},-i \frac{\partial}{\partial x}\right) \frac{\partial T}{\partial t}\left(x^{\prime},-i \frac{\partial}{\partial x}\right)
$$

имеет полный символ $A_{T}$ вида

$$
A_{T}:=\eta I+\left\|b_{i j}^{0}\left(x^{\prime}, \xi\right)\right\|+\sum_{k=1}^{\infty} b^{k}\left(x^{\prime}, \xi\right)
$$

где $b^{k}\left(x^{\prime}, \xi\right)=\left\|b_{i j}^{k}\left(x^{\prime}, \xi\right)\right\|$, и $b^{k}\left(x^{\prime}, \xi\right) \in S^{1-k}\left(\mathbb{R}_{x, \xi}^{2 n} \times[0, T]\right), k \in \mathbb{N}$. В дальнейшем элемент $b_{r+2, r+1}^{1}$ матричного символа $b^{1}\left(x^{\prime}, \xi\right)$ играет важную роль. В работе [3] предполагалось, что в $\mathbb{R}_{x, \xi}^{2 n} \times[0, T] \backslash\{\xi=0\}$ существует $C^{\infty}\left(\mathbb{R}_{x, \xi}^{2 n} \times[0, T] \backslash\{\xi=0\}\right)$ - многообразие $\Sigma$ размерности $2 n$ такое, что

$$
\left.\lambda_{1}\right|_{\Sigma}=\left.\lambda_{2}\right|_{\Sigma}=\left.\lambda_{3}\right|_{\Sigma}, \quad \lambda_{1} \neq \lambda_{2}, \lambda_{3} \neq \lambda_{2}, \lambda_{1} \neq \lambda_{3}
$$

в $\mathbb{R}_{x, \xi}^{2 n} \times[0, T] \backslash(\Sigma \cup\{\xi=0\})$.

Также предполагалось, что функции $\lambda_{1}, \lambda_{2}, \lambda_{3}$ неинволютивны в точках смены кратности $\Sigma$, т.е. скобки Пуассона удовлетворяют условиям

$$
\left.\left\{\eta+\lambda_{1}, \eta+\lambda_{2}\right\}\right|_{\Sigma} \neq 0,\left.\quad\left\{\eta+\lambda_{1}, \eta+\lambda_{3}\right\}\right|_{\Sigma} \neq 0,\left.\quad\left\{\eta+\lambda_{2}, \eta+\lambda_{3}\right\}\right|_{\Sigma} \neq 0,
$$

Работа выполнена при поддержке гранта CONACYT Мексика No. 4297F и гранта SIP-IPN.

(C) В.В. Кучеренко, А. В. Кривко, 2009 
и что

$$
\begin{aligned}
& \inf _{i} \inf _{x^{\prime} \in \mathbb{R}_{x^{\prime}}^{n+1},|\xi|=1}\left\{\left|\lambda_{i}(x, \xi)\right| \geqslant \alpha\right\}>0, \\
& \sup _{i} \sup _{x^{\prime} \in \mathbb{R}_{x^{\prime}}^{n+1},|\xi|=1}\left\{\left|\lambda_{i}(x, \xi)\right| \leqslant \beta\right\}<+\infty .
\end{aligned}
$$

Фактически из условий (2) следует, что

$$
\Sigma=\left\{x^{\prime}, \xi: t=f(x, \xi)\right\}
$$

где

$$
\begin{gathered}
f \in S^{0}\left(\mathbb{R}_{x^{\prime}, \xi}^{2 n+1}\right), \\
\lambda_{j}=a\left(x^{\prime}, \xi\right)+\left(t-f\left(x^{\prime}, \xi\right)\right) \gamma_{j},\left.\quad\left\{\eta+\lambda_{i}, \eta+\lambda_{j}\right\}\right|_{\Sigma}=-\left.\left(\gamma_{i}-\gamma_{j}\right)[1+\{a, f\}]\right|_{\Sigma} \neq 0 .
\end{gathered}
$$

То есть мы имеем $\left.\gamma_{i}\right|_{\Sigma} \neq\left.\gamma_{j}\right|_{\Sigma}$ и $\left.\{a, f\}\right|_{\Sigma} \neq-1$.

В силу условий (2) многообразие $\Sigma$ не содержит траекторий гамильтоновых систем с гамальтонианами $\lambda_{j}\left(x^{\prime}, \xi\right), j=1,2,3$.

Сейчас мы рассматриваем обобщение выше приведенных условий. Предположим, что

$$
\Sigma_{123}:=\left\{x^{\prime}, \xi: \lambda_{1}=\lambda_{2}=\lambda_{3}\right\},
$$

это гладкое $C^{\infty}$-многообразие размерности $(2 n-1)$ в $\mathbb{R}_{x^{\prime}, \xi}^{2 n+1} \backslash\{\xi=0\}$; множества

$$
\Sigma_{i j}:=\left\{x^{\prime}, \xi: \lambda_{i}=\lambda_{j}, 1 \leqslant i, j \leqslant 3, i \neq j\right\},
$$

являются $C^{\infty}$-многообразиями размерности $2 n$ в $\mathbb{R}_{x, \xi}^{2 n} \times[0, T] \backslash\{\xi=0\}$, и что

$$
\left.\left\{\eta+\lambda_{i}, \eta+\lambda_{j}\right\}\right|_{\Sigma_{i j} \cap\{|\xi|=1\}} \neq 0,
$$

для $1 \leqslant i, j \leqslant 3, i \neq j$ (т.е. $\Sigma_{123} \subset \Sigma_{i j}$, в то время как в [3] предполагалось, что $\Sigma_{i j}=$ $\left.\Sigma_{123}=\Sigma\right)$.

Чтобы упростить формулировки ниже приведенных теорем мы предполагаем, что многообразия $\Sigma_{i j} \cap\{|\xi|=1\}$ компактны в $\mathbb{R}_{x, \xi}^{2 n} \times[0, T]$.

Предположим, что волновой фронт начальных данных задачи Коши сконцентрирован на лагранжевом многообразии $\Lambda$ таком, что $\rho\left(\left.\Lambda\right|_{|\xi|=1},\left.\Sigma_{i j}\right|_{|\xi|=1}\right)>0$ для $i, j=1,2,3, i \neq j$. В этом случае [4]-[8] волновой фронт распространяется вдоль траекторий $g_{\lambda_{j}}^{(t, 0)}(x, \xi)$, $(x, \xi) \in \Lambda, j=1,2,3$, гамильтоновых векторных полей $\operatorname{Id} \lambda_{j}$ и их ветвлений. Под ветвлением мы подразумеваем то, что траектория $g_{\lambda_{j}}^{(t, 0)}(x, \xi)$, проходя через точку пересечения $(\bar{t}, \bar{x}, \bar{\xi})$ с многообразиями $\Sigma_{j k}, j \neq k$, генерирует новые ветви-траектории $g_{\lambda_{k}}^{(t, \bar{t})}(\bar{x}, \bar{\xi})$, $t \geqslant \bar{t}$. Кроме того, эти ветви $g_{\lambda_{k}}^{(t, \bar{t})}(\bar{x}, \bar{\xi}), t \geqslant \bar{t}$, в свою очередь, генерируют новые ветви $g_{\lambda_{m}}^{\left(t, \bar{t}_{k}\right)}\left(\bar{x}_{k}, \bar{\xi}_{k}\right), t \geqslant t_{k}, k \neq m$, в точках их пересечения $\left(\bar{t}_{k}, \bar{x}_{k}, \bar{\xi}_{k}\right)$ с многообразиями $\Sigma_{k m}$, $k \neq m$, и так далее.

Из условий (3), (4) следует, что за конечный отрезок времени генерируется конечное число ветвей.

Мы определяем полное ветвление траектории $g_{\lambda_{j}}^{(t, 0)}(x, \xi)$ за время $[0, T]$, как объединение начальной траектории со всеми последовательностями ветвей, сгенерированными ею за время $[0, T]$. Заметим, что из условий (4) следует, что для каждого времени $t_{0}(j, k)$ такого, что $g_{\lambda_{j}}^{\left(t_{0}(j, k), 0\right)}(x, \xi) \in \Sigma_{j k}$, существует $\delta>0$, зависящее от $t_{0}(j, k)$, для которого за время $\left(t_{0}(j, k), t_{0}(j, k)+\delta\right),\left(t_{0}(j, k)-\delta, t_{0}(j, k)\right)$ траектория $g_{\lambda_{j}}^{(t, 0)}(x, \xi)$ не пересекает многообразие $\Sigma_{j k}$.

Объединение волновых фронтов решения задачи Коши $u(t)$ для всех $t \in[0, T]$ содержит полное ветвление траекторий $g_{\lambda_{j}}^{(t, 0)}(x, \xi),(x, \xi) \in \Lambda, j=1,2,3$, за время $T$. 
Когда существуют три характеристических корня, совпадающих на $\Sigma_{123}$, то полное ветвление траекторий, сосредоточенных в некоторой области $\Sigma_{123}$, может содержать периодические циклы или стабильные и нестабильные траектории типа обобщенной логарифмической спирали. Этот эффект не наблюдается в случае, когда совпадают лишь два характеристические корня $\lambda_{i}, \lambda_{j}$, т.е. когда $\Sigma_{123}=\varnothing$.

На рисунке рассмотрен случай размерности $n=1$; символ $\{i, j\}$ обозначает скобку Пуассона $\left\{\lambda_{i}, \lambda_{j}\right\}$ по $\xi, x$. Предполагается, что $\partial \lambda_{j} / \partial t \equiv 0, j=1,2,3$. Определим множества $\Delta_{i j ; k m}^{ \pm \pm}$, как

$$
\Delta_{i j ; k m}^{ \pm \pm}:=\left\{x^{\prime}, \xi: \lambda_{i}-\lambda_{j}<_{(>)} 0, \lambda_{k}-\lambda_{m}<_{(>)} 0\right\}
$$

здесь знак "-" соответствует случаю "<", а знак “+" соответствует случаю ">”.

Предположим, что траектория $g_{\lambda_{2}}^{(t, 0)}(x, \xi)$ начинается в точке $(x, \xi) \in \Delta_{12 ; 31}^{--}=\left\{x^{\prime}, \xi\right.$ : $\left.\lambda_{1}-\lambda_{2}<0, \lambda_{3}-\lambda_{1}<0\right\}$ и выполнены условия

$$
\left.\{1,2\}\right|_{\Sigma_{12}}>0,\left.\quad\{3,2\}\right|_{\Sigma_{32}}<0,\left.\quad\{3,1\}\right|_{\Sigma_{31}}>0 .
$$

На рисунке показано, что полное ветвление траектории $g_{\lambda_{2}}^{(t, 0)}(x, \xi)$ содержит кусочно гладкую траекторию типа логарифмической спирали (на рисунке пунктиром обозначены дополнительные ветви полного ветвления, которые не включены в выделенную траекторию типа логарифмической спирали).

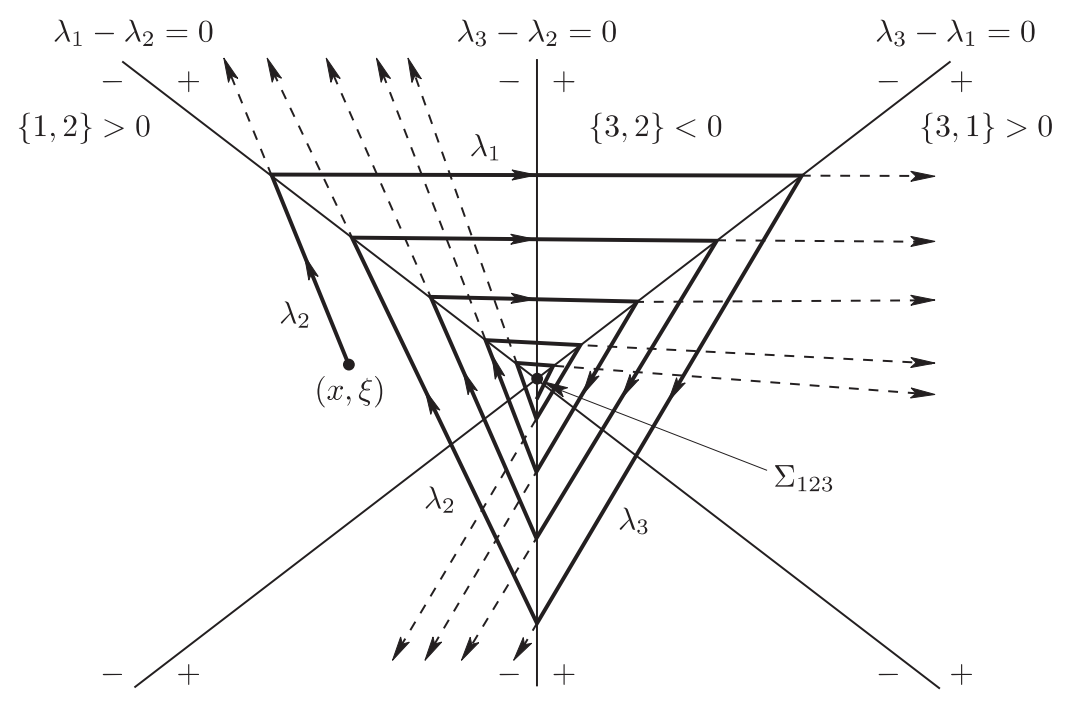

Рис.

Теорема 1. Предположим, что $\operatorname{dim} \Sigma_{123}=2 n-1, \operatorname{dim} \Sigma_{i j}=2 n$, выполнены условия (3), (4). Положим

$$
\Sigma_{123}^{c}:=\Sigma_{123} \cap\left\{\mathbb{R}_{x}^{n} \times\left[C_{1} \leqslant|\xi| \leqslant C_{2}\right]\right\} .
$$

1. Предположим, что в некоторой окрестности $\left(\Sigma_{123}^{c}\right)^{\varepsilon}$ выполнены условия

$$
\left\{\eta+\lambda_{1}, \eta+\lambda_{2}\right\}<0, \quad\left\{\eta+\lambda_{1}, \eta+\lambda_{3}\right\}<0, \quad\left\{\eta+\lambda_{2}, \eta+\lambda_{3}\right\}<0 ;
$$

тогда существует достаточно малое число $\varepsilon_{0}<\varepsilon$ и время $T\left(\varepsilon_{0}\right)>0$ такие, что траектории $g_{\lambda_{j}}^{(t, 0)}(x, \xi), j=1,2,3$, с начальными данными $(x, \xi) \in\left(\Sigma_{123}^{c}\right)^{\varepsilon_{0}}$, и все сгенирированные ими ветви не принадлежат окрестности $\left(\Sigma_{123}^{c}\right)^{\varepsilon_{0}}$ в момент времени $t=T\left(\varepsilon_{0}\right)$.

Траектория $g_{\lambda_{j}}^{(t, 0)}(x, \xi)$ генерирует конечное число ветвей за время $\left[0, T\left(\varepsilon_{0}\right)\right]$. 
2. Предположим, что $n=1$ и в окрестности $\left(\Sigma_{123}^{c}\right)^{\varepsilon}$ выполняются условия

$$
\left\{\lambda_{1}, \lambda_{2}\right\}>0, \quad\left\{\lambda_{3}, \lambda_{2}\right\}<0, \quad\left\{\lambda_{3}, \lambda_{1}\right\}>0, \quad \frac{\partial \lambda_{j}}{\partial t} \equiv 0, \quad j=1,2,3 .
$$

Тогда существует $\varepsilon_{0}<\varepsilon$ такое, что для всех $\varepsilon_{1}, \varepsilon_{2}: 0<\varepsilon_{2}<\varepsilon_{1}<\varepsilon_{0}$, существуют начальные данные $\left(x_{0}, \xi_{0}\right) \in \Delta_{12 ; 31}^{--} \cap\left\{\left(\Sigma_{123}^{c}\right)^{\varepsilon_{1}} \backslash\left(\Sigma_{123}^{c}\right)^{\varepsilon_{2}}\right\}$ такие, что полное ветвление траектории $g_{\lambda_{2}}^{(t, 0)}(x, \xi)$ содержит кусочно гладкую траекторию, состоящую из $N$ элементов вида

$$
\begin{gathered}
\left.g_{\lambda_{2}}^{(t, 0)}\left(x_{0}, \xi_{0}\right) \in \Delta_{12 ; 31}^{--}\right|_{t}, \quad 0 \leqslant t \leqslant t_{1}, \\
\left(x_{1}, \xi_{1}\right):=\left.g_{\lambda_{2}}^{\left(t_{1}, 0\right)}(x, \xi) \in \Sigma_{12}\right|_{t=t_{1}}, \\
\left.g_{\lambda_{1}}^{\left(t, t_{1}\right)}\left(x_{1}, \xi_{1}\right) \in \Delta_{12 ; 31}^{+-}\right|_{t}, \quad t_{1} \leqslant t \leqslant t_{2}, \\
\left(x_{2}, \xi_{2}\right):=\left.g_{\lambda_{1}}^{\left(t_{2}, t_{1}\right)}\left(x_{1}, \xi_{1}\right) \in \Sigma_{31}\right|_{t=t_{2}}, \\
\left.g_{\lambda_{3}}^{\left(t, t_{2}\right)}\left(x_{2}, \xi_{2}\right) \in \Delta_{31 ; 32}^{++}\right|_{t}, \quad t_{2} \leqslant t \leqslant t_{3}, \\
\left(x_{3}, \xi_{3}\right):=\left.g_{\lambda_{3}}^{\left(t_{3}, t_{2}\right)}\left(x_{2}, \xi_{2}\right) \in \Sigma_{32}\right|_{t=t_{3}},
\end{gathered}
$$

u m.d.

$B$ каждом элементе $g_{\lambda_{j}}^{\left(t_{k+1}, t_{k}\right)}\left(x_{k}, \xi_{k}\right)$ этой кусочно гладкой траектории имеем $t_{k+1}$ $t_{k} \geqslant \operatorname{Const}\left(\varepsilon_{2}, \varepsilon_{1}\right) \varepsilon_{2}$, где константа Const $\left(\varepsilon_{2}, \varepsilon_{1}\right)$ не зависит от индекса $k$.

Число элементов $N$ кусочно гладкой траектории удовлетворяет оченке

$$
N \geqslant q\left|\ln \left(\frac{\varepsilon_{1}}{\varepsilon_{2}}\right)\right|,
$$

где константа $q>0$ зависит только от $\varepsilon_{0}$.

Из анализа формально асимптотических решений задачи Коши для гиперболических систем с символом (1) и высокочастотными начальными данными вида $\phi(x) \exp ((i / h) \times$ $\left.S_{0}(x)\right)$ следует, что при распространении вдоль траекторий, определенных в пункте 2 теоремы 1 , амплитуда высокочастотного решения становится порядка $h^{-\sigma N}$, где $\sigma$ зависит от символа младшего порядка $b^{1}(x, \xi)$ [7], [8]. Пусть $\varepsilon_{1}=h^{\delta}, \varepsilon_{2}=h^{\delta / 2}$ для $\delta<1 / 2$ и $\sigma>0$; тогда имеем, что

$$
h^{-\sigma N}=h^{-\sigma q \delta|\ln h| / 2} .
$$

Вышеизложенные рассуждения позволяют доказать, что при некоторых условиях на младшие члены $b_{i j}^{1}$ задача Коши для гиперболической системы с символом (1) является некорректной, т.е. система не строго гиперболична.

Определим $\sigma_{i j}$, как

$$
\sigma_{i j}:=-\left.\frac{i b_{r+2, r+1}^{1} d}{\left\{\eta+\lambda_{j}, \eta+\lambda_{i}\right\}}\right|_{\Sigma_{123}} .
$$

Теорема 2. Предположим, что символ (1) гиперболической системы удовлетворяет условиям (3)-(5), размерность $n=1, \operatorname{dim} \Sigma_{123}=1, \operatorname{dim} \Sigma_{i j}=2 u \partial \lambda_{j} / \partial t \equiv 0$, $j=1,2,3$. Пусть существуют $j: 1 \leqslant j \leqslant r$, такие, что члены нулевого порядка

$$
\begin{gathered}
\left.b_{j, r+1}^{1}\left(x^{\prime}, \xi\right)\right|_{\Sigma_{123} \cap\{|\xi|=1\}} \neq 0,\left.\quad b_{r+1, j}^{1}\left(x^{\prime}, \xi\right)\right|_{\Sigma_{123} \cap\{|\xi|=1\}} \neq 0, \\
\left.\operatorname{Re}\left(\sigma_{32}+3 / 2\right)\right|_{\Sigma_{123} \cap\{|\xi|=1\}}<0 .
\end{gathered}
$$

В этом случае в пространствах $H^{s}$ задача Коши для гиперболических систем с символом (1) является некорректной, т.е. для всех натуральных $k, l, m$ таких, что $k \geqslant m$, 
$l>0$ и для любой константы $C>0$ существует $T>0$ и функция $u_{T}(t)$ со значениями в $C_{0}^{\infty}\left(\mathbb{R}_{x}^{n}\right), u_{T}(t) \in C^{\infty}\left([0, T] ; C_{0}^{\infty}\left(\mathbb{R}_{x}^{n}\right)\right)$, maкие, что

$$
\max _{0 \leqslant t \leqslant T}\left\{\left\|u_{T}(t)\right\|_{H^{k-m}\left(\mathbb{R}_{x}^{n}\right)}\right\}>C \max _{0 \leqslant t \leqslant T}\left\{\left\|L u_{T}(t)\right\|_{H^{l}\left(\mathbb{R}_{x}^{n}\right)}+\left\|u_{T}(0)\right\|_{H^{k}\left(\mathbb{R}_{x}^{n}\right)}\right\} .
$$

Значение Т может быть выбрано произвольно малым.

Из условий теоремы 2 следует существование точки $(\bar{x}, \bar{\xi})$ такой, что $\Sigma_{123}=(\bar{x}, \bar{\xi}) \times$ $[0, T]$, и что полные ветвления траекторий содержат периодические траектории или траектории типа логарифмической спирали.

\section{СПИСОК ЦИТИРОВАННОЙ ЛИТЕРАТУРЫ}

[1] E. Bernardi, A. Bove, Ark. Mat., 43:1 (2005), 113-131. [2] T. Nishitani, J. Anal. Math., 61 (1993), 181-229. [3] А. В. Кривко, В. В. Кучеренко, Докл. РА Н, 412:4 (2007), 451-455. [4] В. В. Кучеренко, Изв. АН СССР. Сер. матем., 38:3 (1974), 625-662. [5] В. Я. Иврий, B. M. Петков, УМH, 29:5 (1974), 3-70. [6] R. B. Melrose, G. A. Uhlmann, Comm. Pure Appl. Math., 32:4 (1979), 483-519. [7] V. V. Kucherenko, A. Kryvko, Stability of Stationary Solutions of Nonlinear Hyperbolic Systems with Multiple Characteristics, Proc. 5th International ISAAC Congress (25-30 July, 2005, University of Catania, Italy). [8] A. Kryvko, Métodos asintóticos para sistemas con características de multiplicidad variable, Thesis, Instituto Politecnico Nacional, Mexico, 2007.

\section{В. В. Кучеренко}

Национальный политехнический институт, Мехико, Мексика E-mail: valeri@esfm.ipn.mx

\section{А. В. Кривко}

Национальный политехнический институт
Поступило

18.02.2008

Исправленный вариант

15.05 .2008 